\title{
Application of the spectral finite element method for elastic wave propagation problems in functionally graded materials
}

\author{
Jonathan Esteban Arroyo Silva ${ }^{1}$ \\ Postgraduate Program in Computational Modeling, Federal University of Juiz de Fora, Brazil \\ Felipe dos Santos Loureiro ${ }^{2}$ \\ Department of Thermal and Fluid Sciences, Federal University of São João del-Rei, Brazil \\ Webe João Mansur ${ }^{3}$ \\ Department of Civil Engineering, COPPE/Federal University of Rio de Janeiro, Brazil
}

\begin{abstract}
This work is concerned with the numerical modeling of elastic wave propagation in a medium constructed with functionally graded materials (FGMs). The FGM is characterized by a gradual change in the material properties over the domain under consideration and its application has been growing in some science and engineering areas. In contrast to layered materials in which effects of reflection and refraction of waves still occur between layers (a situation not always desirable), materials that possess a continuous variation of their properties do not suffer from this drawback. Here, the time-domain elastodynamic equations in FGMs are numerically solved by means of the spectral finite element method based on the Gauss-Lobatto-Legendre points. Owing to the smooth transition of the material properties over the domain makes the SFEM quite suitable for this type of problem since material interfaces are not presented and, therefore, large elements can be easily employed. Furthermore, the SFEM can be viewed as a higher-order finite element method (FEM) and has been receiving great popularity for owning the FEM geometric flexibility in creating meshes among other numerical features such as less dispersion errors and mass lumping. At the end of the paper, a numerical example considering a FGM is presented, and the results are compared with those furnished by the the equivalent homogeneous and layered bi-material models to illustrate the difference of the models.
\end{abstract}

Keywords. elastic waves, spectral element, functionally graded materials

\section{Introduction}

In the study of wave propagation problems, the low-order FEM is not usually preferred due to its low accuracy, requiring a fine mesh to yield accurate results. Hence, although the FEM has flexibility in creating meshes for any kind of geometry, finite difference methods are still widely used for solving the elastic and acoustic wave equations especially in some

\footnotetext{
${ }^{1}$ jeas560@gmail.com

${ }^{2}$ felipe.loureiro@ufjf.edu.br

${ }^{3}$ webe@coc.ufrj.br
} 
areas such as the geophysics [4]. Recently, higher-order methods like the SFEM have been introduced to simulate these phenomena [7]. The SFEM has some of the FEM properties, and it is able to achieve the expected accuracy using few grid points per wavelength [6], and differs from the classical higher-order FEM that presents some numerical problems such as the occurrence of high oscillations, known as the Runge phenomenon [2].

Functionally graded materials are widely used in contemporary science due to their distinct properties [8] and, thus, the development and study of numerical methods for the solution of problems that adopt such type of material is of great importance. Hence, the behavior of these materials in the context of elastic wave propagation problems is investigated here using the SFEM and then compared with other material models.

\section{Spectral finite element method in functionally graded ma- terials}

This section presents the equations used to model elastic wave propagation phenomena in $2 \mathrm{D}$, the system of equations obtained with the classic semi-discrete FEM formulation and the main differences when the SFEM is employed, and the graded finite elements formulation to model functionally graded materials.

\subsection{Governing equations and the semi-discrete formulation}

Let $\Omega \subset \mathbb{R}^{2}$ be a bounded domain and $I=(0, T] \subset \mathbb{R}^{+}$be the time domain of the analysis, the elastic wave propagation problems are modeled by the following equations in index notation:

$$
\rho \ddot{u}_{i}-\sigma_{i j, j}=b_{i} \text { in } \Omega \times I
$$

where $u_{i}: \Omega \times I \rightarrow \mathbb{R}$ and $\sigma_{i j}: \Omega \times I \rightarrow \mathbb{R}$ stand for the displacement and Cauchy stress tensor components, respectively, and $\rho: \Omega \rightarrow \mathbb{R}_{+}$is the mass density while $b_{i}: \Omega \times I \rightarrow \mathbb{R}$ is the given body force components. In order to have a well-posed problem, it is necessary to impose initial and boundary conditions. Thus, considering the partition $\partial \Omega=\Gamma=$ $\Gamma_{D_{i}} \cup \Gamma_{N_{i}}$ with $\Gamma_{D_{i}} \cap \Gamma_{N_{i}}=\emptyset$ the boundary conditions can be written as:

$$
u_{i}=\bar{u}_{i} \text { on } \Gamma_{D_{i}} \times I, \quad \sigma_{i j} n_{j}=\bar{t}_{i} \text { on } \Gamma_{N_{i}} \times I
$$

where $\bar{u}_{i}: \Gamma_{D_{i}} \times I \rightarrow \mathbb{R}$ are prescribed displacements and $\bar{t}_{i}: \Gamma_{N_{i}} \times I \rightarrow \mathbb{R}$ are prescribed tractions with $n_{j}$ being the unit outward normal vector components. To complete the statement of the problem, the prescribed initial conditions are given by:

$$
u_{i}=u_{0 i} \text { in } \Omega \text { for } t=0, \quad \dot{u}_{i}=v_{0 i} \text { in } \Omega \text { for } t=0
$$

When the classic semi-discrete finite element formulation is applied to above equations [1], one obtains a system of ordinary differential equations, i.e.:

$$
\mathbf{M} \ddot{\mathbf{U}}+\mathbf{K} \dot{\mathbf{U}}=\mathbf{F}
$$


where $\mathbf{M} \in \mathbb{R}^{n q \times n q}$ and $\mathbf{K} \in \mathbb{R}^{n q \times n q}$ are the global mass and stiffness matrices, respectively, and $\mathbf{F}: I \rightarrow \mathbb{R}^{n q}$ is the external force vector. Vectors $\mathbf{U}: I \rightarrow \mathbb{R}^{n q}$ and $\ddot{\mathbf{U}}: I \rightarrow \mathbb{R}^{n q}$ represent respectively, nodal displacements and accelerations with $n q$ being the number of equations. In this work the solution of Eqs. (1)-(3) is restricted to linear problems, i.e., $\sigma_{i j}=C_{i j k l} u_{k, l}$ where $C_{i j k l}: \Omega \rightarrow \mathbb{R}$ is the elasticity tensor components.

\section{$2.2 \quad$ Legendre spectral element}

In the FEM, the original physical domain $\Omega$ is discretized into nel non-overlapping element domains $\Omega_{e}$, and the unknown fields are approximated in the spatial domain taking into account basis functions $N_{i}$. The SFEM follows a similar approach but it is characterized by the use of high-order Lagrange polynomials of degree $p$ in each direction (see Figure 1). In this way, over each $2 D$ element, basis functions $N_{i}$ are constructed taking into account the tensor product of Lagrange polynomials that pass through the $p+1$ GaussLobatto-Legendre (GLL) points (see Figure 1b). As a result, the 2D quadrilateral spectral element is formed by a total of $(p+1)^{2}$ nodes as depicted in Figure 2. In Figure 1 one can also see the difference between the use of classical equidistant points and the GLL points in which for the former high oscillations near the end points appear, which is known as Runge phenomenon as previously commented.
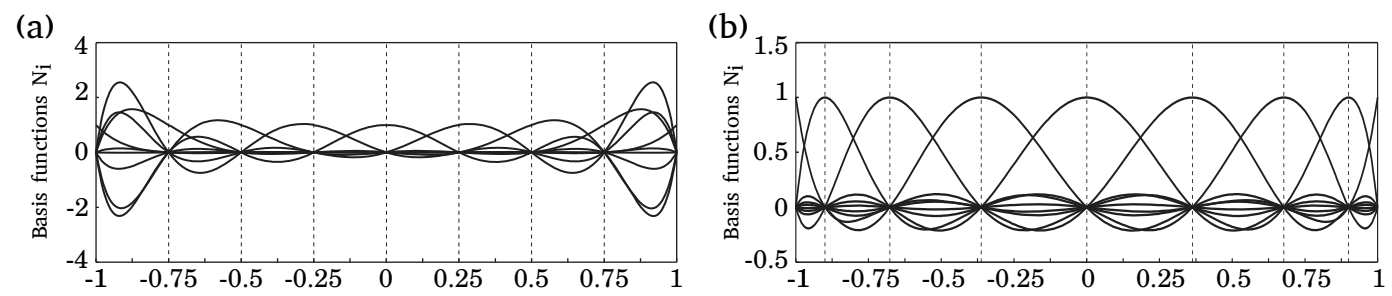

Figure 1: Comparison among the different 1D shape functions considering (a) equidistant points; and (b) GLL points.

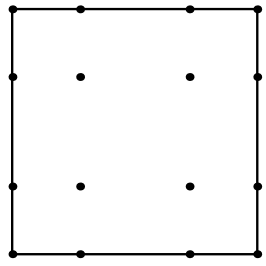

(a)

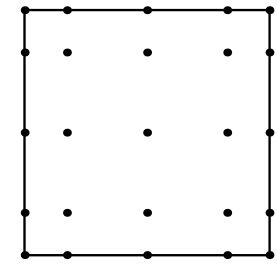

(b)

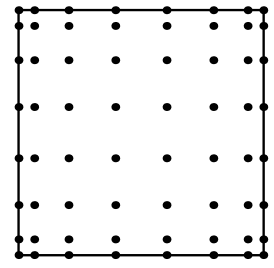

(c)

Figure 2: Different kinds of 2D-SFEs with respect to the polynomial order $p$ : (a) $3 \times 3$; (b) $4 \times 4$; (c) $7 \times 7$.

One major advantage of the SFEM in the context of wave problems lies in the fact that only 4 or 5 points per minimum wavelength are required when working with a polynomial degree between $p=5$ and $p=8$, resulting in meshes with a smaller number of elements and 
nodes when compared to the classic 4-noded quadrilateral element wich requires between 15 to 20 points.

\subsection{Graded finite elements}

There are different ways to deal with the material non-homogeneity, the most common way consists in the use of homogeneous elements with constant material properties at the element level. Zhang and Paulino [8] developed the so-called Generalized Isoparametric Formulation that consists in incorporating the material property variation at the element by employing the same shape functions adopted to interpolate the unknown displacement field. In their study, the use of this strategy results in smoother and more accurate solutions when conpared to the homogeneous element strategy. In this way, the interpolation for the material properties can written as

$$
E=\sum_{i=1}^{n n e} N_{i} E_{i}, \quad \nu=\sum_{i=1}^{n n e} N_{i} \nu_{i}, \quad \rho=\sum_{i=1}^{n n e} N_{i} \rho_{i}
$$

where $N_{i}$ are the basis functions, nne is the number of nodes per element, $E$ is the Young's Modulus, $\nu$ is the Poisson's ratio and $\rho$ is the mass density. From a practical point of view the use of such approximations is quite straightforward in the sense that material properties are generally known from experimental data or standard physical tables.

The use of non-homogeneity at the element level results in slightly different matrices from those obtained for constant materials, i.e.:

$$
\mathbf{M}^{e}=\int_{\Omega^{e}} \mathbf{N}^{T} \rho(\mathbf{x}) \mathbf{N} d \Omega^{e}, \quad \mathbf{K}^{e}=\int_{\Omega^{e}} \mathbf{B}^{T} \mathbf{C}(\mathbf{x}) \mathbf{B} d \Omega^{e}
$$

where $\mathbf{B}$ denotes the matrix of the spatial derivatives of the basis functions containing in the matrix $\mathbf{N}$ and $\mathbf{C}$ is the constitutive material matrix; note that both $\mathbf{C}$ and $\rho$ depend on the global coordinates. Likewise, wave velocities inside the element are no longer constants, e.g. the dilatational wave speed $C_{d}$ is now expressed as

$$
C_{d}^{2}(\mathbf{x})=\frac{E(\mathbf{x})(1-\nu(\mathbf{x}))}{(1+\nu(\mathbf{x}))(1-2 \nu(\mathbf{x})) \rho(\mathbf{x})}
$$

It is worth mentioning that the size of the time step can be readily estimated by invoking the Courant condition [1], which provides an upper limit for the size of the time step $\Delta t$ employed in classical explicit schemes. Thus, considering the highest velocity in the model defined as $C_{d}^{h}=\max \left\{C_{d}(\mathbf{x}), \mathbf{x} \in \Omega\right\}$, one obtains:

$$
n_{C}=\frac{C_{d}^{h} \Delta t}{h}
$$

where $h$ is the smallest distance between two consecutive nodes and $n_{C}$ is the Courant number. In practice, for polynomials approximations of order $p=5$ or $p=8$, the Courant number must be of the order of 0.60 [5]. 
Additionally, to simplify the computer implementation, only a linear variation for the material properties along either $x$ or $y$ direction is here considered, resulting in the below expressions (along the $y$ direction which is adopted in the example):

$$
E(y)=E_{1}+\left(E_{2}-E_{1}\right) y / L, \nu(y)=\nu_{1}+\left(\nu_{2}-\nu_{1}\right) y / L, \quad \rho(y)=\rho_{1}+\left(\rho_{2}-\rho_{1}\right) y / L
$$

where subscripts 1 and 2 denote the two end points $y=0$ and $y=L$, respectively. For the equivalent homogeneous material one considers:

$$
\bar{E}=\frac{1}{L} \int E(y) d y, \quad \bar{\nu}=\frac{1}{L} \int \nu(y) d y, \quad \bar{\rho}=\frac{1}{L} \int \rho(y) d y
$$

which are defined as the equivalent material constants.

Finally, it is important to highlight that if the mass matrix is evaluated taking into account the GLL quadrature a diagonal matrix is obtained.

\section{Numerical example and discussion}

The numerical example presented here consists of showing the different behaviors of the wave propagation under the equivalent homogeneous material, bi-material and FGM. Furthermore, a comparison between the results obtained using the SFEM and the FEM are presented and discussed. In Figure 3 one can see a sketch of the elastic models used

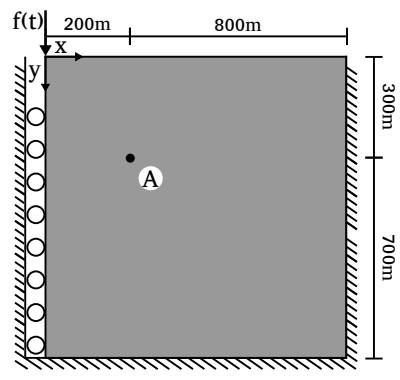

(a)

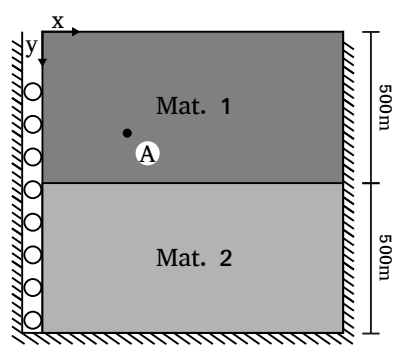

(b)

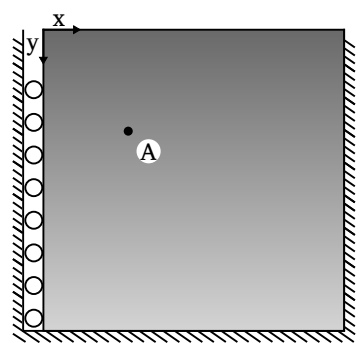

(c)

Figure 3: Elastic models with different material profiles: (a)Equivalent Homogeneous, (b)Bi-material, (c) FGM.

here, where in the top left corner a vertical point load is applied and its time variation is defined as $f(t)=10^{3}\left(1-2 \pi^{2} f_{c}^{2}\left(t-t_{0}\right)^{2}\right) e^{-\left(\pi f_{c}\left(t-t_{0}\right)\right)^{2}}$, where $t_{0}=0.1 s$ with $f_{c}=12.5 \mathrm{~Hz}$ being the central frequency.

A mesh composed of 400 spectral elements of order $p=7$ with an element length $l_{e}=50$ is adopted to the SFEM, whereas 10000 four-noded quadrilateral elements with $l_{e}=10$ are considered in the FEM. The Newmark scheme with a time-step size $\Delta t=5 \times 10^{-4} s$ is used for both methods. The physical properties of the three models are shown in Table 1 .

Figure 4 shows the results obtained at point A (see Figure 3); at the left graphic it is readily seen the difference of the three models; all of them are solved with a very fine FE mesh (such a result will be used as a reference solution). 
Table 1: Material properties

\begin{tabular}{|c|c|c|c|c|c|c|}
\hline Material & $\nu$ & $\rho\left(\mathrm{kg} / \mathrm{m}^{3}\right)$ & $E\left(\mathrm{~N} / \mathrm{m}^{2}\right)$ & $c_{d}(\mathrm{~m} / \mathrm{s})$ & $c_{s}(\mathrm{~m} / \mathrm{s})$ & $c_{r}(\mathrm{~m} / \mathrm{s})$ \\
\hline Homo. & 0.265 & 4000 & $3 \times 10^{9}$ & 3045 & 1722 & 1584 \\
\hline Mat. 1 & 0.31 & 5000 & $2 \times 10^{9}$ & 2355 & 1236 & 1146 \\
\hline Mat. 2 & 0.22 & 3000 & $4 \times 10^{9}$ & 3902 & 2338 & 2132 \\
\hline
\end{tabular}

Furthermore, the solution of the FGM model is more similar to that of the equiv. homogeneous model than the solution of the bi-material model which suffers with the wave reflection effects on the materials interface. In the next two graphics, it is possible to see that the solution obtained by the SFEM is more accurate than that obtained by the FEM which adopts a larger amount of elements.
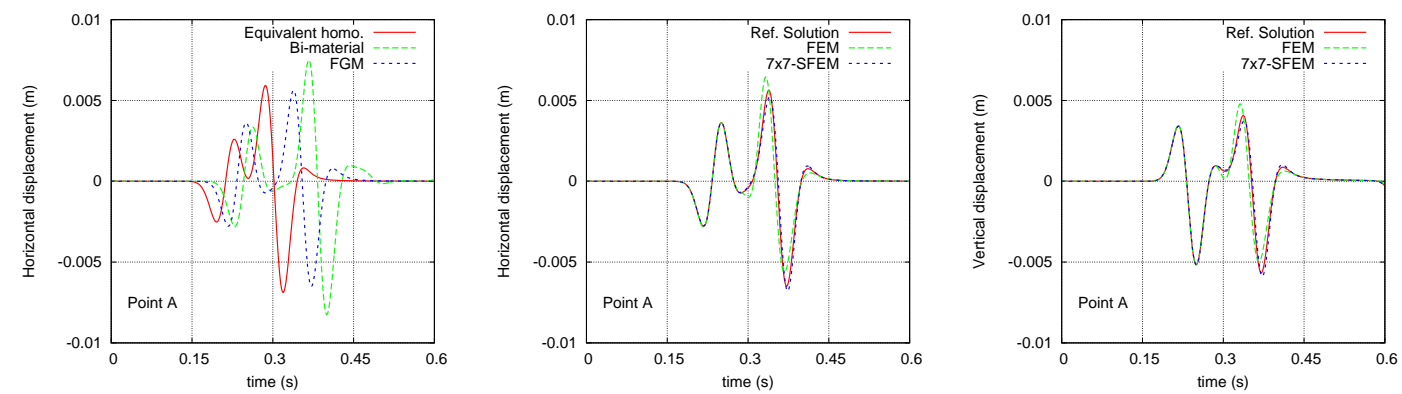

Figure 4: Comparison of the numerical results at point A: the horizontal displacement in different models (left); and the horizontal displacement (middle) and vertical displacement (right) with the different methods.

Differently from the FGM and equiv. homogeneous models, in the bi-material one refraction and reflection are clearly observed when the P-wave crosses the interface between the materials (see Figure 5b). Moreover, unlike the homogeneous model, in the FGM model (Fig. 5c) it is observed that the wave front (P-wave or S-wave) propagates faster in the $\mathrm{y}$-direction than in the $\mathrm{x}$-direction caused by the increase of the vertical velocity.

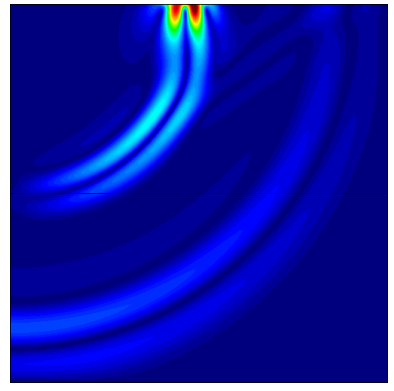

(a)

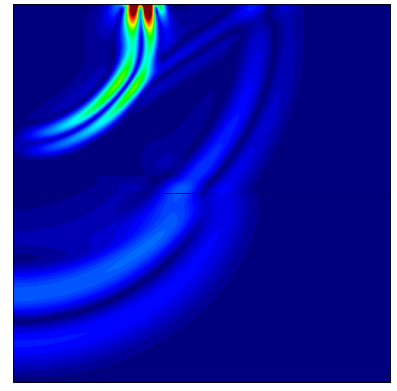

(b)

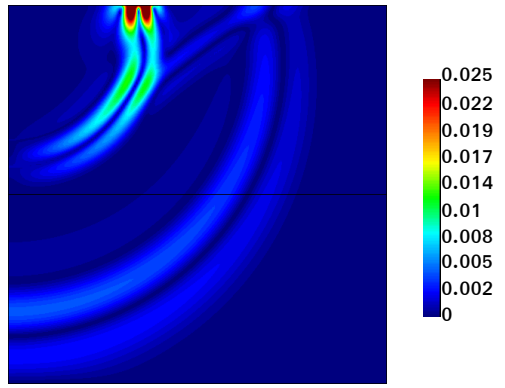

(c)

Figure 5: Snapshot of the $\|\mathbf{u}\|$ at $\mathrm{t}=0.4 \mathrm{~s}$ for the models: (a)Equivalent homogeneous, (b)Bi-material, (c)FGM. 


\section{Conclusions}

In a great deal of cases the reflection and refraction presented in the Bi-material model are not desired. As presented in this work, functionally graded materials can be efficiently adopted to avoid this issue since the material properties are continuous throughout the domain under consideration. The elastodynamic equations with this type of material were easily implemented numerically and the use of the SFEM seems to be quite appropriated because models do not present an interface between the materials and therefore distorted elements are avoided. Furthermore, because of its low dispersion, only 4 or 5 nodes per minimum wavelength need to be considered in the discretization, as previously commented. Thus, a smaller system of equations but with a higher sparsity profile is generated when compared to the FEM. However, one possible solution for this issue is to condense internal nodes as can be seen in [3].

\section{Acknowledgement}

The financial support of CNPQ, FAPEMIG and UFJF is gratefully acknowledged.

\section{References}

[1] K. J. Bathe, Finite element procedures, Prentice-Hall, (1996).

[2] J. P. Boyd, Chebyshev and Fourier Spectral Methods: Second Revised Edition, Dover Publications, (2001).

[3] S. Dong, Z. Yosibash, A parallel spectral element method for dynamic threedimensional nonlinear elasticity problems, Comp. \& Str., vol. 87, 59-72, (2009).

[4] K. R. Kelly and K. J. Marfurt, Numerical modeling of seismic wave propagation, SEG, (1990).

[5] D. Komatitsch and J. Tromp, Introduction to the spectral element method for threedimensional seismic wave propagation, GJI, vol. 139, 806-822, (1999).

[6] D. Komatitsch, J. P. Vilotte, R. Vai, J. M. Castillo-Covarrubias and F. J. SanchezSesma, The spectral element method for elastic wave equations: aplication to $2 \mathrm{~d}$ and 3d seismic problems, IJNME, vol. 45, 1139-1164, (1999).

[7] D. Komatitsch, Q. Liu, J. Tromp, P. Suss, C. Stidham and J. H. Shaw, Simulations of ground motion in the Los Angeles basin based upon the spectral-element method, BSSA, vol. 94, 187-206, (2004).

[8] Z. Zhang and G. H. Paulino, Wave propagation and dynamic analysis of smoothly graded heterogeneous continua using graded finite elements, IJSS, vol. 44, 3601-3626, (2007). 